\title{
Cardiac Protection by Oral Sodium Thiosulfate in a Rat Model of L-NNA-Induced Heart Disease
}

\author{
Isabel T. N. Nguyen ${ }^{1}$, Lucas M. Wiggenhauser ${ }^{2}$, Marian Bulthuis ${ }^{2}$, Jan-Luuk Hillebrands ${ }^{2}$, \\ Martin Feelisch ${ }^{3}$, Marianne C. Verhaar ${ }^{1}$, Harry van Goor $^{2 \dagger}$ and Jaap A. Joles ${ }^{1 \dagger *}$ \\ ${ }^{1}$ Department of Nephrology and Hypertension, University Medical Center Utrecht, Utrecht, Netherlands, ${ }^{2}$ Department of \\ Pathology and Medical Biology, University Medical Center Groningen and University of Groningen, Groningen, Netherlands, \\ ${ }^{3}$ Clinical and Experimental Sciences, Faculty of Medicine, Southampton General Hospital and Institute for Life Sciences, University \\ of Southampton, Southampton, United Kingdom
}

OPEN ACCESS

Edited by:

Andreas Papapetropoulos, National and Kapodistrian University

of Athens, Greece

Reviewed by:

Vincenzo Brancaleone, University of Basilicata, Italy

Ahmed F. El-Yazbi, American University of Beirut,

Lebanon

*Correspondence: Jaap A. Joles

J.A.Joles@umcutrecht.nl

tThese authors have contributed equally to this work and share last authorship

Specialty section:

This article was submitted to Cardiovascular and Smooth Muscle

Pharmacology,

a section of the journal

Frontiers in Pharmacology

Received: 08 January 2021

Accepted: 11 March 2021

Published: 15 April 2021

Citation:

Nguyen ITN, Wiggenhauser $L M$,

Bulthuis $M$, Hillebrands $J-L$, Feelisch M, Verhaar MC, van Goor $H$ and Joles JA (2021) Cardiac Protection

by Oral Sodium Thiosulfate in a Rat

Model of L-NNA-Induced

Heart Disease.

Front. Pharmacol. 12:650968.

doi: 10.3389/fphar.2021.650968
Hypertension contributes to cardiac damage and remodeling. Despite the availability of renin-angiotensin system inhibitors and other antihypertensive therapies, some patients still develop heart failure. Novel therapeutic approaches are required that are effective and without major adverse effects. Sodium Thiosulfate (STS), a reversible oxidation product of hydrogen sulfide $\left(\mathrm{H}_{2} \mathrm{~S}\right)$, is a promising pharmacological entity with vasodilator and antioxidant potential that is clinically approved for the treatment of calciphylaxis and cyanide poisoning. We hypothesized that Sodium Thiosulfate improves cardiac disease in an experimental hypertension model and sought to investigate its cardioprotective effects by direct comparison to the ACE-inhibitor lisinopril, alone and in combination, using a rat model of chronic nitric oxide (NO) deficiency. Systemic nitric oxide production was inhibited in Sprague Dawley rats by administering $\mathrm{N}$ - $\omega$-nitro-L-arginine (L-NNA) with the food for three weeks, leading to progressive hypertension, cardiac dysfunction and remodeling. We observed that STS, orally administered via the drinking water, ameliorated L-NNA-induced heart disease. Treatment with STS for two weeks ameliorated hypertension and improved systolic function, left ventricular hypertrophy, cardiac fibrosis and oxidative stress, without causing metabolic acidosis as is sometimes observed following parenteral administration of this drug. STS and lisinopril had similar protective effects that were not additive when combined. Our findings indicate that oral intervention with a $\mathrm{H}_{2} \mathrm{~S}$ donor such as STS has cardioprotective properties without noticeable side effects.

Keywords: hydrogen sulfide, hypertension, ACE-inhibitor, cardiovascular disease, nitric oxide

\section{INTRODUCTION}

Cardiovascular diseases represent a major global health problem and are a leading cause of morbidity and mortality worldwide. Hypertension is one of the most important risk factors for the development of various cardiac ailments, such as coronary heart disease, left ventricular hypertrophy, valvular heart disease and heart failure (Kjeldsen, 2018). The hemodynamic stress imposed by hypertension can induce cardiac remodeling involving cardiomyocyte hypertrophy, interstitial inflammation, fibrosis and microvascular rarefaction (Drazner, 2011). These maladaptive changes can eventually lead to left ventricular dysfunction and heart failure. Inhibition of the renin-angiotensin system 
(RAS) has shown clear benefit in this setting by reversing cardiac remodeling. However, despite widespread therapeutic use of RAS inhibitors and other antihypertensive therapies, patients still develop heart failure (Koitabashi and Kass, 2011). This demonstrates the ongoing need for novel therapeutic approaches that are effective and without major adverse effects.

Hydrogen sulfide $\left(\mathrm{H}_{2} \mathrm{~S}\right)$ is the third endogenous gaseous transmitter, in addition to nitric oxide (NO) and carbon monoxide (CO) (Wang, 2010), and has many biological properties, including anti-oxidative, anti-apoptotic, proangiogenic, vasodilating properties and endothelial NO synthase (eNOS) modulating activities. At physiological $\mathrm{pH}$, most $\mathrm{H}_{2} \mathrm{~S}$ exists in the form of the hydrosulfide anion $\left(\mathrm{HS}^{-}\right.$). As a powerful nucleophile and reducing agent it has the potential to modulate a wide range of pathophysiological processes (Liu et al., 2012; Pan et al., 2014). $\mathrm{H}_{2} \mathrm{~S}$ is endogenously produced from the metabolism of L-cysteine by cystathionine $B$-synthase (CBS) and cystathionine $\gamma$-lyase (CSE) and from D-cysteine by 3mercaptopyruvate sulfurtransferase (3-MST) (Fu et al., 2012). In the presence of oxygen, $\mathrm{HS}^{-}$can undergo oxidation to form sulfoxide species such as sulfite, sulfate, thiosulfate, polythionates, persulfides and polysulfides (Powell et al., 2018). In human physiology, $\mathrm{H}_{2} \mathrm{~S}$ can be regenerated from per/polysulfides, sulfite and thiosulfate, but not from sulfate (Olson, 2011; Perridon et al., 2016).

Lower levels of endogenous $\mathrm{H}_{2} \mathrm{~S}$ in plasma and tissues have been observed in various cardiovascular disease states, including heart failure, myocardial ischemia and atherosclerosis (Li et al., 2016; Pan et al., 2017), and exogenous treatment with $\mathrm{H}_{2} \mathrm{~S}$ donors has been shown to be cardioprotective in various experimental models of cardiac injury. Although most of these investigations focused on acute myocardial protection, there are some studies on the protective effects of $\mathrm{H}_{2} \mathrm{~S}$ in experimental models of chronic heart failure. In experimental models, $\mathrm{H}_{2} \mathrm{~S}$ is often delivered via inhalation or by parenteral administration of simple inorganic salts such as sodium hydrosulfide (NaHS) and sodium sulfide $\left(\mathrm{Na}_{2} \mathrm{~S}\right)$. A potential alternative exists in the form of sodium thiosulfate (STS, $\mathrm{Na}_{2} \mathrm{~S}_{2} \mathrm{O}_{3}$ ), an endogenous metabolite that is produced during mitochondrial oxidation of $\mathrm{H}_{2} \mathrm{~S}$. In humans, short-term therapeutic intravenous and oral use of high concentrations of STS has been proven safe for the treatment of calciphylaxis (Singh et al., 2011), providing an opportunity for utilization of $\mathrm{H}_{2} \mathrm{~S}$-related therapies in other clinical settings. Recently, the intravenous administration of STS up to a dosage of $15 \mathrm{~g}$ was found to be well-tolerated and safe in patients with acute coronary syndrome (de Koning et al., 2020). Vasodilating and antioxidant properties have been attributed to STS (Sen et al., 2008). These beneficial effects would seem to make STS an attractive therapeutic candidate to tackle the cardiovascular alterations associated with hypertension.

Recently, we demonstrated that both orally administered and intraperitoneal injected STS improved renal function and damage in rat models of hypertension (Snijder et al., 2014; Nguyen et al., 2020). However, to the best of our knowledge, the effects of STS supplementation, compared to RAS inhibition, on cardiac dysfunction have not yet been investigated. In the present study, we sought to investigate the cardioprotective effects of orally administered STS in comparison to the angiotensinconverting enzyme (ACE) inhibitor lisinopril, alone and in combination, in a rat model of chronic NO deficiency. $\mathrm{N}-\omega$ Nitro-L-Arginine (L-NNA) was used to induce hypertension in conscious rats, as previously performed at our laboratory (Verhagen et al., 1999). Chronic inhibition of all NOS isoforms including eNOS, by either L-NNA or its methylester L-NAME (N- $\omega$-Nitro-L-Arginine methylester), leads to dosedependent severe hypertension, cardiac dysfunction and remodeling (Verhagen et al., 1999; Kobayashi et al., 2000; Biwer et al., 2013; Jin et al., 2017). This rat model was chosen as it is a reliable and reproducible model that develops hypertension within a short period of time, allowing us to evaluate the use of STS in hypertensive heart disease. Pharmacological inhibition of endogenous NO production in this model has the added advantage of simplifying the experimental landscape by removing an additional layer of complexity otherwise arising due to the chemical interactions between $\mathrm{H}_{2} \mathrm{~S}$ and $\mathrm{NO}$ (Cortese-Krott et al., 2015), which can considerably complicate the interpretation of experimental results. We hypothesized that STS improves cardiac function, hypertrophy and damage in L-NNA-induced heart disease.

\section{MATERIALS AND METHODS}

\section{Animals}

Male Sprague-Dawley rats (300-350 g, Envigo, Venray, the Netherlands) were cohoused in a climate-controlled facility with a 12-h light-dark cycle. Rats had access to water and standard rat chow (CRM-E; Special Diet Services, Witham, Essex, United Kingdom) ad libitum. All procedures were approved by the Animal Ethics Committee of the University of Utrecht (CCD: AVD1150020171484) and were in accordance with the Dutch Codes of Practice for the Care and Use of Animals for Scientific Purposes.

\section{Study Design}

Following transportation, rats were allowed one week of acclimatization before experimental uses. After a baseline period, global NOS activity was inhibited by administering L-NNA (40 mg/kg/day; N5501-25G, Sigma-Aldrich, Zwijndrecht, the Netherlands) with the food for three weeks. After one week of inhibition of NOS, animals were divided into four groups 1) L-NNA only $(\mathrm{N}=8)$, 2) L-NNA with STS (2 g/kg/ day; 217247-500G, Sigma-Aldrich) administered via the drinking water for two weeks (L-NNA + STS; N = 7), 3) L-NNA with lisinopril ( $1 \mathrm{mg} / \mathrm{kg} /$ day; PHR1143-1G, Sigma-Aldrich) mixed with the food (L-NNA + lisinopril; $\mathrm{N}=5$ ) and 4) L-NNA with both lisinopril in the food and STS in the drinking water (L-NNA + STS + lisinopril; $\mathrm{N}=5$ ). The dose of STS was previously determined in a pilot study (Nguyen et al., 2020). The dose of lisinopril chosen for this study is very low in comparison to most other studies with lisinopril in models of heart disease, in order to allow observation of additional effects of STS. Starting at baseline, systolic blood pressure (SBP) was 
measured via tail-cuff plethysmography, and body weight was monitored every three days. Echocardiography was performed at baseline and after three weeks of L-NNA treatment. Reported baseline values for SBP and echocardiographic variables belong to the rats from the L-NNA group without any treatment and were measured before L-NNA treatment was initiated. At the end of the experiments, blood gas analysis and morphological analysis of the heart were performed in all four groups.

\section{Echocardiographic Evaluation}

Transthoracic echocardiography was performed with a digital ultrasound machine (Sonos 5500, Philips Research, Eindhoven, the Netherlands) and a $15 \mathrm{MHz}$ linear array transducer (Hewlett Packard, Palo Alto, CA). Rats were anesthetized with isoflurane (3\% for induction and $2-2.5 \%$ for maintenance) and placed in supine position on a heating pad. Two-dimensional B-mode cine loops were recorded in the parasternal long-axis and the midpapillary short-axis views. Left ventricular (LV) volume was calculated with the area-length method at end-diastole and end-systole in the parasternal long axis view (Bongartz et al., 2010). Cardiac output and ejection fraction were then calculated from these LV volumes. Systolic and diastolic wall thickness and cavity dimensions were recorded in M-mode in the short-axis view. Images from apical 4-chamber view were acquired to evaluate LV filling and diastolic function. Mitral flow velocity tracings were obtained with pulsed-wave Doppler. Peak early E (E wave) and A (A-wave) filling velocities were measured. Tissue Doppler imaging as used to obtain early (e') diastolic velocity at the medial mitral annulus. For evaluation of diastolic function, the ratio of peak velocity early (E) over late (A) mitral inflow and the ratio $\mathrm{E}$ over $\mathrm{e}^{\prime}$ was calculated. The recordings were coded for blinded analysis and analyzed offline using the system's standard operating software. Variables were measured in at least three heart beats at enddiastole and end-systole.

\section{Blood Gas Analysis}

Blood gas variables from the femoral artery were analyzed on a hand-held blood gas analyzer (i-STAT, Abbot, Hoofddorp, the Netherlands).

\section{Urine Collection for Oxidative Stress Measurement}

For collection of $24 \mathrm{~h}$-urines, rats were individually housed in metabolic cages without food but with access to drinking water supplemented with $2 \%$ glucose. Urinary excretion of lipid peroxides was measured as thiobarbituric acid reactive substances (TBARS) using a commercial kit (TBARS assay CAYM10009055-96, Cayman Chemical, Ann Arbor, MI) according to manufacturer's instructions.

\section{Cardiac Histology}

To investigate cardiac fibrosis, Masson's Trichrome staining was used on paraffin-embedded cardiac sections. Subsequently, cardiac sections were scanned using a Hamamatsu
Nanozoomer HT 2.0 (Hamamatsu Photonics, Japan). The extent of collagen deposition was determined using the Aperio Image Scope positive pixel count analysis V9.1 algorithm. The ratio between the fibrotic surface area and the total cardiac surface area was calculated. Histopathological analysis was performed in a blinded fashion.

To investigate cardiomyocyte cross-sectional area, formalinfixed paraffin-embedded (FFPE) cardiac sections were deparaffinized, rehydrated and antigen retrieval in boiling Citrate buffer $(10 \mathrm{mM}, \mathrm{pH} 6.0)$ was performed. Cardiac sections were incubated with Wheat-Germ Agglutinin (WGA)FITC (1:200, L4895-2 mg, Sigma) conjugate for $60 \mathrm{~min}$ at room temperature. Nuclei were counterstained with DAPI $(4,6-$ diamino-2-phenylindole dilactate (Invitrogen, Oregon United States) 1:40.000 in Fluoromount (DAKO Glostrup, Denmark). Stitched high-magnification (40x) scans were captured with TissueFAXS acquisition software on a Zeiss AxioObserver Z1 fluorescence microscope (TissueGnostics, Vienna, Austria). Subendocardial areas with cardiomyocytes in cross-sectional orientation were selected for analysis in ImageJ (version 1.53c; National Institutes of Health, Bethesda, MD). Cell boundaries were identified using Contrast Limited Adaptive Histogram Equalization (CLAHE), thresholding and binary options and cross-sectional cardiomyocyte area was measured in at least 1500 cells per sample.

\section{Statistical Analysis}

Data were analyzed using GraphPad 7.0 Software (GraphPad, San Diego, CA). Data are presented as mean \pm standard error of the mean (SEM). For longitudinal measurements, a twoway ANOVA for repeated measures was performed, followed by Bonferroni post-hoc test. Baseline values were only compared to the L-NNA group using a paired $t$-test and comparisons between the four groups were done using a oneway ANOVA, followed by Dunnett's multiple comparison test.

\section{RESULTS}

\section{STS Treatment Decreases Systolic Blood Pressure}

Chronic systemic NO deficiency was induced by administering L-NNA with the food (40 mg/kg/day) for three weeks (Nguyen et al., 2020). Administration for two weeks of either STS ( $2 \mathrm{~g} / \mathrm{kg} /$ day) via the drinking water, the ACE-inhibitor lisinopril ( $1 \mathrm{mg} / \mathrm{kg} /$ day) via the food or their combination was started one week after initiation of NOS inhibition. As we previously reported (Nguyen et al., 2020), the administration of L-NNA rapidly induced progressive hypertension (Figure 1A) and treatment with STS was able to lower SBP measured by tail-cuff plethysmography. Treatment with lisinopril showed an antihypertensive effect, although at this low dose, SBP did not return to baseline levels. STS plus lisinopril did not further improve SBP compared to lisinopril alone. Body weights (BW) did not differ significantly between groups (Figure 1B). 

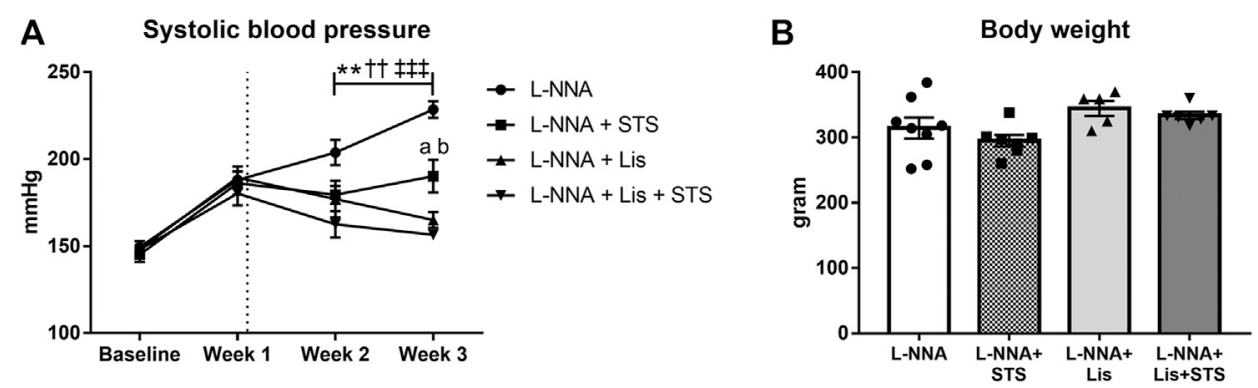

FIGURE 1 |STS administration decreases systolic blood pressure. (A) Longitudinal measurements of systolic blood pressure and (B) body weight at the end of the experimental protocol are shown for the four groups. $\mathrm{N}=5-8$. ${ }^{*} \mathrm{~L}-\mathrm{NNA}$ vs. L-NNA + STS, ' $\mathrm{L}-\mathrm{NNA}$ vs. L-NNA + Lis, ${ }^{\ddagger} \mathrm{L}-\mathrm{NNA}$ vs. L-NNA + Lis + STS, ${ }^{\mathrm{L}} \mathrm{L}-\mathrm{NNA}+\mathrm{Lis}$ vs. L-NNA + STS, ' ${ }^{2}-N N A+$ STS vs. L-NNA + Lis + STS. Two symbols $p<0.01$, three symbols $p<0.001$. Data previously published (Nguyen et al., 2020).
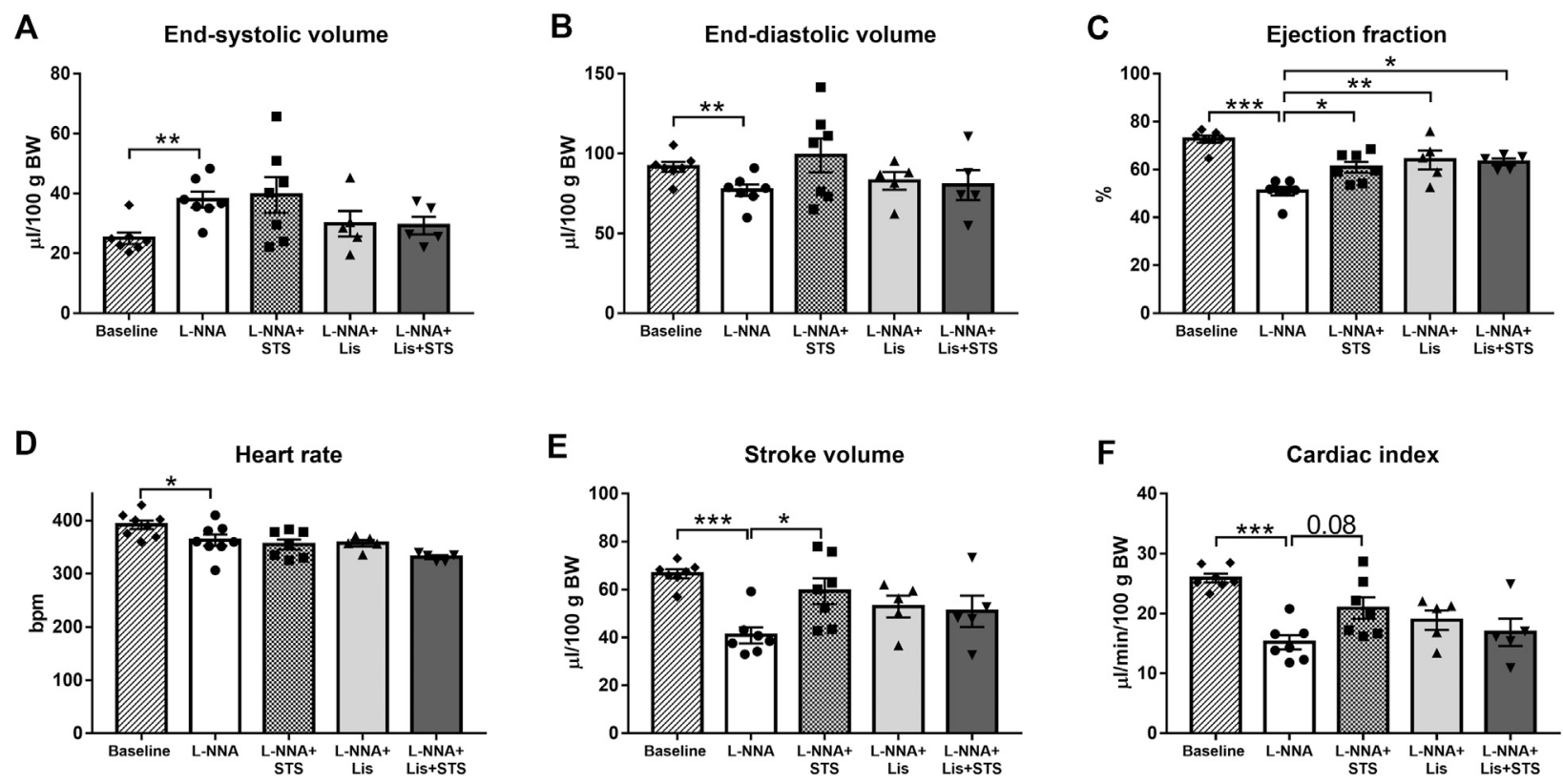

FIGURE 2 | Treatment with STS improves systolic function. (A) End-systolic volume and (B) end-diastolic volume were measured to calculate (C) ejection fraction. (D) Heart rate and (E) stroke volume were used to calculate (F) cardiac index in the four groups. $\mathbf{N}=5-8 .{ }^{*} p<0.05,{ }^{* *} p<0.01,{ }^{* \star *} p<0.001$.

\section{Oral Administration of STS Improves Systolic Function}

Echocardiography was performed at baseline, before L-NNA administration was started, and after three weeks of L-NNA administration and two weeks of STS and/or lisinopril treatment. Chronic global NOS inhibition caused an increase in end-systolic volume $(37.9 \pm 2.6$ vs. $25.1 \pm 2.0 \mu \mathrm{L} / 100 \mathrm{~g} \mathrm{BW}$; Figure $2 \mathrm{~A}$ ) and a decrease in end-diastolic volume (77.0 \pm 3.6 vs. $91.7 \pm 3.1 \mu \mathrm{L} / 100 \mathrm{~g} \mathrm{BW}$; Figure 2B) and ejection fraction (72.2 \pm $1.5 \%$ vs. $51.0 \pm 1.7 \%$; Figure $2 \mathrm{C}$ ) compared to baseline values. Treatment with STS improved ejection fraction compared to L-NNA only $(60.9 \pm 2.3 \%)$. Lisinopril alone as well as the combination of STS and lisinopril also improved ejection fraction, however the combination therapy did not cause further improvement compared to either lisinopril or STS alone. Administration of L-NNA further caused a decline in heart rate $(362 \pm 11 \mathrm{bpm}$ vs. $391 \pm 8 \mathrm{bpm})$, stroke volume $(41.0 \pm 3.3$ vs. $66.6 \pm 1.9 \mu \mathrm{L} / 100 \mathrm{~g} \mathrm{BW})$ and cardiac index $(15.2 \pm 1.2$ vs. $25.9 \pm 0.7 \mu \mathrm{L} / \mathrm{min} / 100 \mathrm{~g} \mathrm{BW})$ compared to baseline levels (Figure 2D-F). Oral administration of STS increased stroke volume $(59.3 \pm 5.4 \mu \mathrm{L} / 100 \mathrm{~g} \mathrm{BW})$ and tended to improve cardiac index $(20.9 \pm 1.8 \mu \mathrm{L} / \mathrm{min} / 100 \mathrm{~g} \mathrm{BW} ; p=0.08)$. As compared to STS, no differences were observed upon treatment with lisinopril or the combination therapy.

\section{L-NNA Administration did Not Affect Diastolic Function}

Pulsed-wave Doppler and Tissue Doppler echocardiography were used to evaluate diastolic function. Chronic NO 

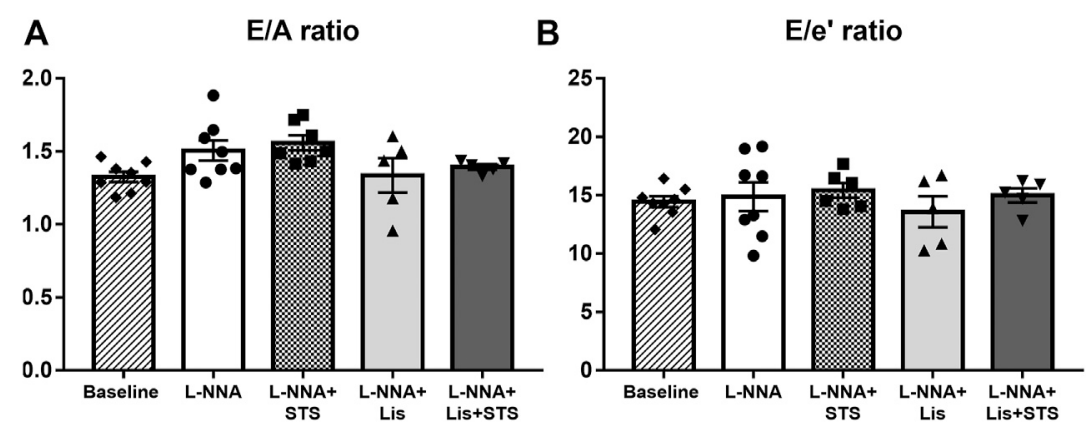

FIGURE 3 | Diastolic function was not affected in L-NNA-induced hypertensive rats. Pulsed-wave and Tissue Doppler imaging were used to calculate (A) E/A ratio and (B) E/e' ratio to evaluate diastolic function. $\mathrm{N}=5-8$.
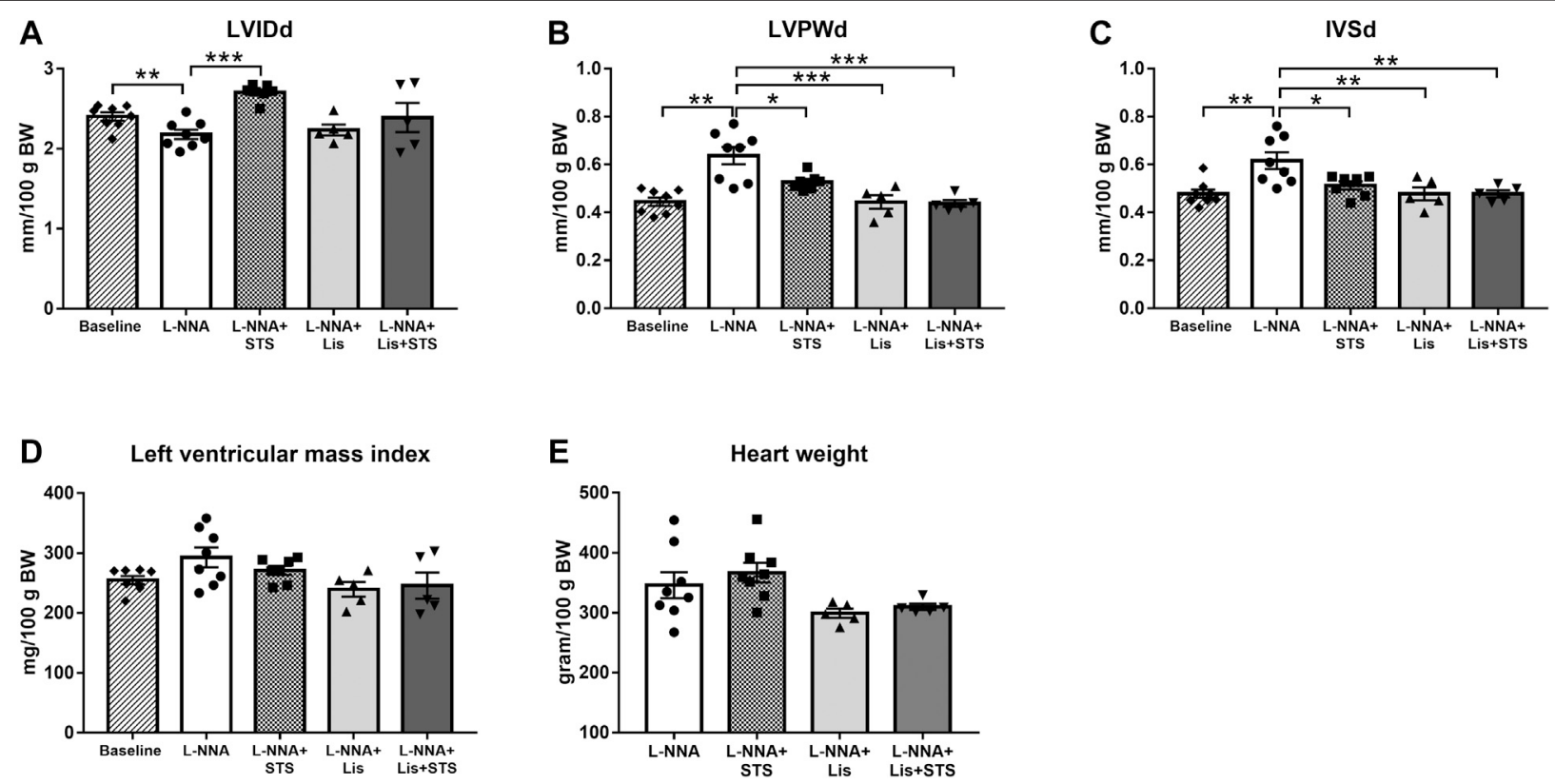

FIGURE 4 | Treatment with STS improved left ventricular posterior and septal wall hypertrophy. (A) Left ventricular internal diameter (LVIDd), (B) left ventricular posterior wall thickness (LVPWd) and (C) interventricular septal wall thickness (IVSd) during diastole were measured via echocardiography. (D) Left ventricular mass index was calculated from these variables and $(\mathbf{E})$ heart weight was measured at the end of the protocol. $N=5-8 .{ }^{*} p<0.05,{ }^{* *} p<0.01,{ }^{* \star *} p<0.001$.

deficiency did not affect E/A ratio (Figure $\mathbf{3 A}$ ) and E/e' ratio (Figure $3 \mathbf{B}$ ). Treatment with STS, lisinopril or the combination of STS and lisinopril also did not affect these variables.

\section{STS Administration Improved Left Ventricular Posterior and Septal Wall Hypertrophy}

M-mode echocardiography in the short axis at the level of the papillary muscles was performed in order to assess cardiac wall dimensions. Left ventricular internal diameter during diastole (LVIDd) was lowered after three weeks of L-NNA administration $(2.2 \pm 0.06$ vs. $2.4 \pm 0.05 \mathrm{~mm} / 100 \mathrm{~g} \mathrm{BW})$
(Figure 4A). L-NNA further increased the thickness of the left ventricular posterior wall (LVPWd; $0.64 \pm 0.04$ vs. $0.45 \pm$ $0.02 \mathrm{~mm} / 100 \mathrm{~g} \mathrm{BW}$ ) and intraventricular septal wall during diastole (IVSd; $0.62 \pm 0.04$ vs. $0.48 \pm 0.02 \mathrm{~mm} / 100 \mathrm{~g} \mathrm{BW}$ ) (Figures 4B,C), indicative of left ventricular wall hypertrophy. Treatment with STS for two weeks increased LVIDd $(2.7 \pm 0.04 \mathrm{~mm} / 100 \mathrm{~g} \mathrm{BW})$ and lowered LVPWd and IVSd $(0.53 \pm 0.01$ and $0.51 \pm 0.02 \mathrm{~mm} / 100 \mathrm{~g}$ BW, respectively). Lisinopril also reduced LVPWd and IVSd thickness $(0.44 \pm 0.03$ and $0.48 \pm 0.03 \mathrm{~mm} / 100 \mathrm{~g} \mathrm{BW})$ similar to the combination therapy $(0.44 \pm 0.01$ and $0.48 \pm 0.02 \mathrm{~mm} / 100 \mathrm{~g} \mathrm{BW})$. Calculated left ventricular mass index and measured wet heart weight after three weeks of L-NNA administration was not different between groups (Figures 4D,E). 

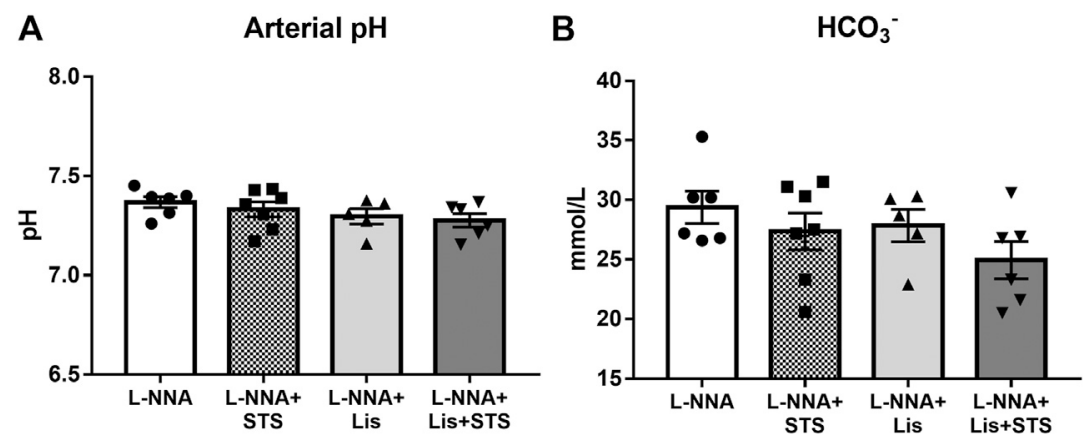

FIGURE 5 | Oral treatment of STS does not lead to metabolic acidosis. (A) $\mathrm{pH}$ and $\mathbf{( B )} \mathrm{HCO}_{3}{ }^{-}$were measured in arterial blood via blood gas analysis at the end of the experimental protocol. $\mathrm{N}=5-7$.

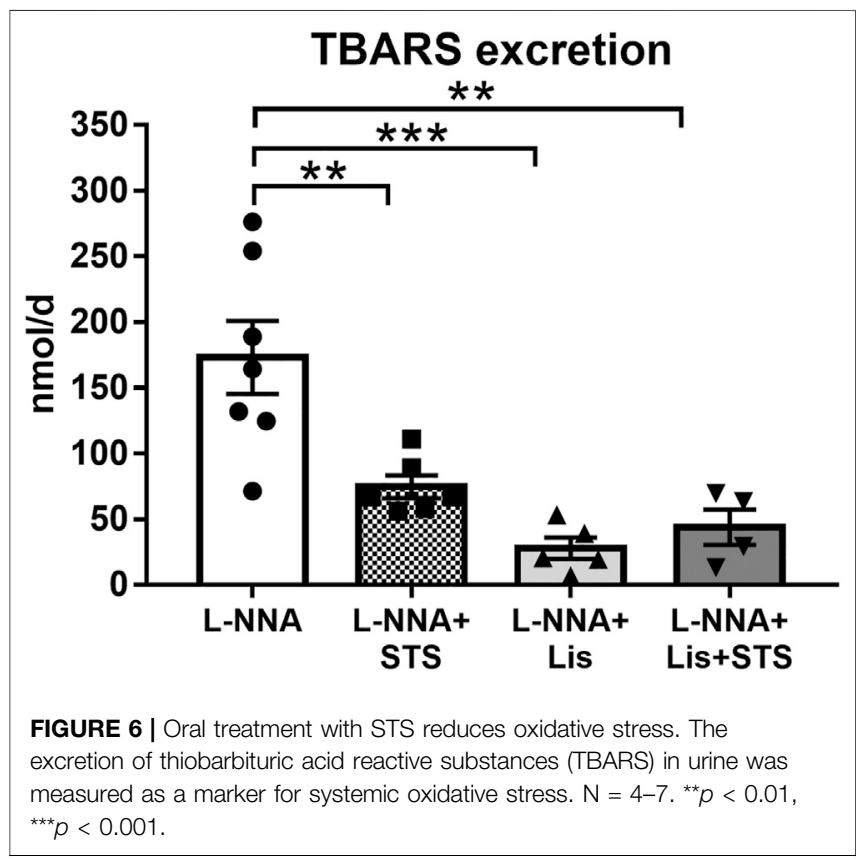

\section{Oral Administration of STS for Two Weeks Does Not Lead to Metabolic Acidosis}

After three weeks of chronic inhibition of NOS, blood gas analysis was performed on arterial blood samples to determine arterial $\mathrm{pH}$ (Figure 5A) and bicarbonate (Figure 5B) to assess the effect of STS on these variables. No differences were observed in arterial $\mathrm{pH}$ and bicarbonate between treatment groups, with or without STS.

\section{STS Treatment Reduced Oxidative Stress}

Systemic oxidative stress, as measured by the urinary excretion of TBARS, was lowered after treatment with STS $(74.7 \pm 8.7$ vs. $173.1 \pm 27.6 \mathrm{nmol} /$ day) (Figure 6). Lisinopril also reduced TBARS excretion, as did the combination therapy $(27.9 \pm 8.5$ and $43.9 \pm 13.5 \mathrm{nmol} /$ day).

\section{STS Administration Improved Cardiac Remodeling}

Cardiac sections were stained with Masson Trichrome to determine collagen deposition following three weeks of L-NNA administration and two weeks of intervention (Figure 7A). A reduction in fibrosis was found after treatment with STS or lisinopril $(0.094 \pm 0.01$ and $0.066 \pm 0.01$ vs. $0.15 \pm$ $0.01 \%$ ) compared to L-NNA alone (Figure 7B). Combination of STS with lisinopril did not further improve fibrosis compared to STS or lisinopril alone. Cardiac sections were stained with WheatGerm Agglutinin -FITC to assess cardiomyocyte cross-sectional area (Figure 8A). There was a trend toward a lower cardiomyocyte size in the STS- and STS + Lisinopril-treated rats ( $p=0.07$ and $p=0.08$, respectively) compared to L-NNA only (Figure 8B). At this low dose of lisinopril, no difference was observed compared to the L-NNA group.

\section{DISCUSSION}

The major finding of our study is that oral administration of STS via the drinking water ameliorates L-NNA-induced heart disease to the same extent as ACE inhibition. Treatment with STS for two weeks improved systolic function, left ventricular wall hypertrophy, cardiac fibrosis and systemic oxidative stress. No signs of metabolic acidosis were observed following oral administration of STS. This indicates that oral intervention with the $\mathrm{H}_{2} \mathrm{~S}$ donor STS is safe and has promising cardioprotective effects.

\section{Oral STS Attenuated L-NNA-Induced Hypertension}

The reduction in blood pressure by orally administered STS observed in this study (Nguyen et al., 2020) is in line with previous findings with parenteral treatment with NaHS. Early studies on $\mathrm{H}_{2} \mathrm{~S}$ delivery showed that the use of aqueous NaHS in rat aortic rings induced marked relaxation revealing the vasorelaxant properties of $\mathrm{H}_{2} \mathrm{~S}$ (Zhao et al., 2001). Furthermore, administration of NaHS lowered blood pressure 

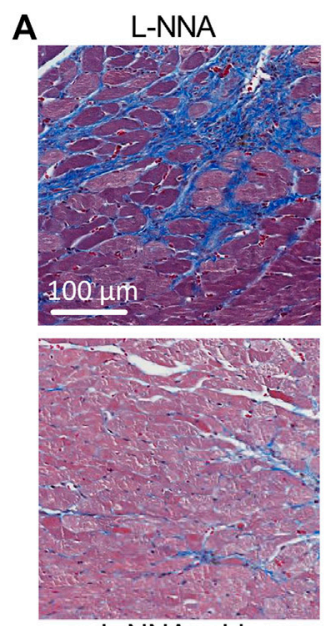

L-NNA + Lis
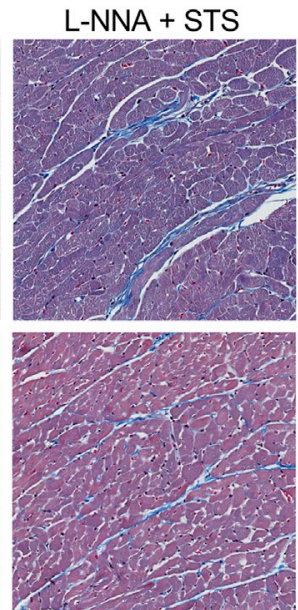

L-NNA + STS + LiS

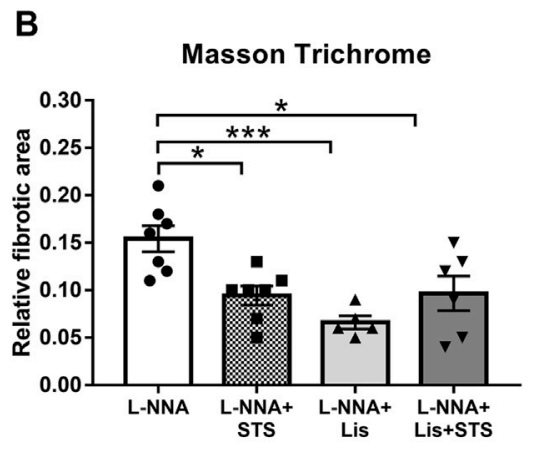

FIGURE 7 | Treatment with STS reduced cardiac fibrosis. Masson Trichrome stained cardiac sections were used to visualize collagen deposition. (A) Representative images and (B) quantification of the fibrotic area in the four groups. $\mathrm{N}=5-7 .{ }^{*} p<0.05,{ }^{\star * *} p<0.001$.

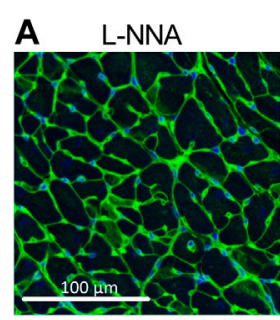

L-NNA + STS

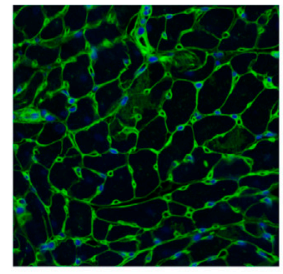

L-NNA + Lis

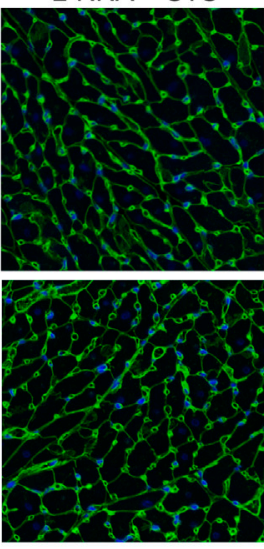

L-NNA + STS + LiS

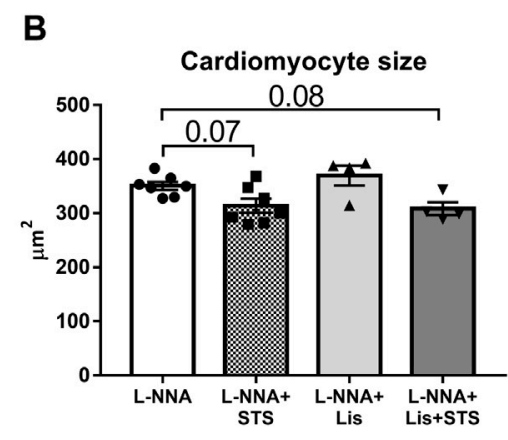

FIGURE 8 | Treatment with STS tends to lower cardiomyocyte hypertrophy. Cardiac sections were stained with Wheat-Germ Agglutinin-FITC to investigate cardiomyocyte size. (A) Representative images and (B) quantification of the cardiomyocyte cross-sectional area in the four groups. $\mathrm{N}=4-7$.

in experimental models of hypertension (Zhong et al., 2003; Yan et al., 2004; Ahmad et al., 2014; Snijder et al., 2014; Xiao et al., 2016). We observed a similar blood pressure reduction after lisinopril administration and no additive effect with the combination therapy. It is well-known that inhibition of the RAS system is able to normalize arterial blood pressure in hypertensive heart disease both in spontaneously hypertensive rats (Brilla et al., 1996) and in rats with L-NNA-induced hypertension (Verhagen et al., 2000). Additionally, ACEinhibitors inhibit the degradation of bradykinin, thereby increasing their blood pressure lowering effects (Grafe et al., 1993). The mechanism of blood pressure reduction observed with STS in our study remains unknown. STS may react via various thiol reactions involving transsulfuration enzymes with cysteine to produce $\mathrm{H}_{2} \mathrm{~S}$ in the smooth muscle cells (Sowers and Hayden,
2010). It has been shown that $\mathrm{H}_{2} \mathrm{~S}$ can affect the RAS system directly, by inhibiting the activity of renin and ACE, by interfering with zinc at the active site of ACE (Laggner et al., 2007; Lu et al., 2010). This suggests that STS and lisinopril might act via the same pathway to lower the blood pressure, which would also explain why the antihypertensive effects were not additive.

Other proposed mechanisms for the vasodilating effects of $\mathrm{H}_{2} \mathrm{~S}$ include the activation of $\mathrm{K}_{\mathrm{ATP}}$ sensitive channels through cysteine-S-sulfhydration, induction of Keap1/Nrf2 signaling and augmentation of endogenous NO signaling (Jiang et al., 2010; Mustafa et al., 2011; Huang et al., 2016; Nabeebaccus and Shah, 2018). $\mathrm{H}_{2} \mathrm{~S}$ also prevents the inactivation of cyclic adenosine monophosphate and cyclic guanosine monophosphate by nonselective inhibition of phosphodiesterases (Bucci et al., 
2010). Furthermore, STS may recouple endothelial NOS via restoration of tetrahydrobiopterin to its fully reduced state, thereby restoring bioavailable NO (Whiteman and Moore, 2009). However, since our model relies on the systemic inhibition of endogenous NOS production, we believe that these mechanisms cannot explain the effects observed in the current study.

In addition, oxidative stress can contribute to the development of hypertension by reducing endothelial NO bioavailability (Schulz et al., 2011). Both STS and lisinopril treatment had anti-oxidant effects, evidenced by lower TBARS excretion. Our findings suggest that the antihypertensive effect exerted via STS or lisinopril may be mediated via common pathways associated with oxidative stress and that treatment with STS might be beneficial for patients with hypertensive heart disease that respond poorly to RAS inhibition. However, for the same reasons as alluded to above, this mechanism is unlikely to be mediated via an enhancement of endogenous $\mathrm{NO}$ availability. It is possible that the observed reductions of lipid oxidation products in urine were the result of acute antiinflammatory effects or due to a modulation of cellular mitochondrial function; dedicated experiments would be required to confirm or exclude these possibilities, which was beyond the purpose of the current study.

\section{Oral STS Improves Systolic Function and Cardiac Remodeling}

Growing evidence shows that $\mathrm{H}_{2} \mathrm{~S}$ improves cardiac function and remodeling in different cardiovascular disorders, such as myocardial ischemia reperfusion injury, myocardial infarction, cardiac arrhythmia and heart failure (Shen et al., 2015). Treatment with NaHS attenuated cardiac dysfunction, evidenced by increased ejection fraction, and reduced cardiac fibrosis in rats with myocardial infarction and heart failure (Pan et al., 2014; Abdelmonem et al., 2019). Treatment with STS for six weeks prevented the development of ventricular contractile dysfunction, thickening of the left ventricular posterior wall and collagen deposition in mice with chronic heart failure (Sen et al., 2008). Intraperitoneal injections with STS reduced the degree of cardiac hypertrophy and fibrosis in angiotensin IIinduced hypertensive rats (Snijder et al., 2015). STS might trigger the regulation of matrix metalloproteinases in the failing heart, thereby attenuating collagen deposition in the extracellular matrix (Sen et al., 2008).

In our study, treatment with STS was started one week after LNNA-induced hypertension was established, whereas in previous studies STS treatment is often initiated together with disease induction. Here we observed that orally administered STS improved systolic function and reduced cardiac fibrosis. The low dose of the ACE-inhibitor lisinopril, purposely chosen to allow observation of additional effects of STS, improved cardiac function and remodeling similar to treatment with STS. Additionally, intervention with STS on top of lisinopril did not perform better compared to either STS or lisinopril alone. Lisinopril has been shown to enhance collagen degradation by activation of tissue matrix metalloproteinase 1 in spontaneously hypertensive rats (Brilla et al., 1996). As for hypertension, this suggests that the antifibrotic response exerted via STS or lisinopril is mediated via common pathways.

It has been reported that NaHS treatment ameliorated L-NAME-induced cardiac dysfunction and remodeling via activation of the Akt/eNOS/NO pathway mediated by $\mathrm{K}_{\mathrm{ATP}}$ channels (Jin et al., 2017). $\mathrm{H}_{2} \mathrm{~S}$ markedly prevented the development of cardiac fibrosis and decreased the collagen content in the cardiac tissue by inhibiting the activity of intracardiac angiotensin II (Huang et al., 2012). In addition, the suppression of inflammation, the reduction of cardiac apoptosis from oxidative stress and improving mitochondrial derangements are likely to have antifibrotic roles under hypertensive conditions (Wang et al., 2011; Kang et al., 2020). Antihypertensive effects of $\mathrm{H}_{2} \mathrm{~S}$, via modulation of channel activities and the cGMP/PKG pathways, may also contribute to the suppression of fibrosis caused by hypertension (Meng et al., 2015).

Chronic NO inhibition by L-NAME has been shown to induce cardiomyocyte hypertrophy in rats (Sanada et al., 2001). In our study orally administered STS tended to decrease cardiomyocyte cross-sectional area. Parenteral treatment with STS or NaHS attenuated cardiomyocyte hypertrophy in angiotensin II induced hypertensive rats (Snijder et al., 2015). Although ACE-inhibition effectively reduces cardiomyocyte size (Tamura et al., 2000), the low dosage of lisinopril used in this study did not decrease cardiomyocyte cross-sectional area. Our findings suggest that oral intervention with STS might be beneficial in cardiovascular disorders associated with a reduced left ventricular contractile function and cardiac remodeling.

\section{Metabolic Acidosis}

For the administration of $\mathrm{H}_{2} \mathrm{~S}$ in experimental models, soluble sulfide salts are often used while occasionally gaseous $\mathrm{H}_{2} \mathrm{~S}$ has been administered. In clinical practice, STS has been approved in two indications: 1) together with sodium nitrite, as antidote for cyanide intoxications, and 2) for the short-term treatment of calcific uremic arteriolopathy in patients with end-stage kidney disease. In both indications, intravenous infusion of STS is the preferred route of administration. In some cases, such patients developed metabolic acidosis with an anion gap following parenteral infusion with STS (Rein et al., 2014; Hunt and Ryder, 2018). In our study we showed that STS, when orally administered via the drinking water, did not lead to metabolic acidosis, suggesting that oral treatment with STS might be a more advantageous route of administration. It should be noted that kidney function is only reduced by about $50 \%$ in this model (Verhagen et al., 1999). We cannot exclude that even oral STS may lead to metabolic acidosis when kidney function is more severely compromised.

\section{Oral Treatment With STS Reduces Oxidative Stress}

Previously we have shown that various conditions associated with systemic oxidative stress are accompanied by increased urinary 
TBARS excretion, namely chronic kidney disease induced by subtotal nephrectomy (Papazova et al., 2014), chronic hypertension in spontaneously hypertensive rats (Racasan et al., 2004; Koeners et al., 2011), and dietary hypercholesterolemia (Attia et al., 2003). Importantly, crosstransplantation of healthy and diseased kidneys in healthy recipients or rats with long-standing chronic kidney disease convincingly showed that urinary TBARS excretion in these animals represents systemic but not intrarenal oxidative stress (Papazova et al., 2018). The production of reactive oxygen species causes endothelial dysfunction and can therefore also contribute to the development of hypertension (Schulz et al., 2011). $\mathrm{H}_{2} \mathrm{~S}$ is a known antioxidant by increasing the levels of glutathione and directly scavenging reactive oxygen species (Kimura et al., 2010). In addition to its vasodilator action, eNOS-derived NO is also a potent antioxidant with cardioprotective effects (Erkens et al., 2018), and we cannot exclude that L-NNA induced global NOS inhibition in our model may have sensitized the heart to other antioxidants effects. Parenteral treatment with STS has been shown to reduce the levels of oxidative stress in angiotensin II-induced hypertensive rats (Snijder et al., 2015). STS might act as an antioxidant by scavenging superoxide in the myocardial tissue, thereby helping to rescue the failing heart (Sen et al., 2008). Here we showed that oral treatment with STS reduces systemic oxidative stress as evidenced by a lower TBARS excretion. These antioxidant effects of STS, in addition to its vasodilating properties, may have also contributed to the cardioprotection observed in this study.

\section{CONCLUSION}

Orally administered STS improves systolic function, and ameliorates hypertension, left ventricular hypertrophy, fibrosis and systemic oxidative stress, all to the same extent as ACE inhibition. These findings indicate that oral treatment with STS has marked cardioprotective properties and shows therapeutic potential in hypertensive heart disease.

\section{REFERENCES}

Abdelmonem, M., Shahin, N. N., Rashed, L. A., Amin, H. A. A., Shamaa, A. A., and Shaheen, A. A. (2019). Hydrogen sulfide enhances the effectiveness of mesenchymal stem cell therapy in rats with heart failure: in vitro preconditioning versus in vivo co-delivery. Biomed. Pharmacother. 112, 108584. doi:10.1016/j.biopha.2019.01.045

Ahmad, F. U. D., Sattar, M. A., Rathore, H. A., Tan, Y. C., Akhtar, S., Jin, O. H., et al. (2014). Hydrogen sulphide and tempol treatments improve the blood pressure and renal excretory responses in spontaneously hypertensive rats. Ren. Fail. 36, 598-605. doi:10.3109/0886022X.2014.882218

Attia, D. M., Goldschmeding, R., Attia, M. A., Boer, P., Koomans, H. A., and Joles, J. A. (2003). Male gender increases sensitivity to renal injury in response to cholesterol loading. Am. J. Physiol.-Renal Physiol. 284, F718-F726. doi:10.1152/ ajprenal.00009.2002

Biwer, L. A., Broderick, T. L., Xu, H., Carroll, C., and Hale, T. M. (2013). Protection against L-NAME-induced reduction in cardiac output persists even after cessation of angiotensin-converting enzyme inhibitor treatment. Acta Physiol. 207, 156-165. doi:10.1111/j.1748-1716.2012.02474.x

\section{DATA AVAILABILITY STATEMENT}

The raw data supporting the conclusions of this article will be made available by the authors, without undue reservation.

\section{ETHICS STATEMENT}

The animal study was reviewed and approved by the Animal Ethics Committee of the University of Utrecht (CCD: AVD1150020171484) and was in accordance with the Dutch Codes of Practice for the Care and Use of Animals for Scientific Purposes.

\section{AUTHOR CONTRIBUTIONS}

IN, LW, and MB contributed to the acquisition, analysis and interpretation of data for the work. J-LH, MV, HG and JJ acquired funding. All authors contributed to drafting the manuscript and revising it critically for important intellectual content.

\section{FUNDING}

This work was supported by the Dutch Kidney Foundation (13OI114 and 170116) and by a grant from the Netherlands CardioVascular Research Initiative: an initiative with support of the Dutch Heart Foundation (CVON2014-11 (RECONNECT)). Part of the work has been performed at the UMCG Imaging and Microscopy Center (UMIC), which is sponsored by NWO-grant 40-00506-98-9021 (TissueFAXS).

\section{ACKNOWLEDGMENTS}

We thank Petra de Bree and Melanie Nieuwenhuijzen-Van de Kaa for their technical advice and assistance.

Bongartz, L. G., Braam, B., Verhaar, M. C., Cramer, M. J., Goldschmeding, R., Gaillard, C. A., et al. (2010). Transient nitric oxide reduction induces permanent cardiac systolic dysfunction and worsens kidney damage in rats with chronic kidney disease. Am. J. Physiol.-Regul., Integr. Comp. Physiol. 298, R815-R823. doi:10.1152/ajpregu.00727.2009

Brilla, C. G., Matsubara, L., and Weber, K. T. (1996). Advanced hypertensive heart disease in spontaneously hypertensive rats. Hypertension 28, 269-275. doi:10. 1161/01.hyp.28.2.269

Bucci, M., Papapetropoulos, A., Vellecco, V., Zhou, Z., Pyriochou, A., Roussos, C., et al. (2010). Hydrogen sulfide is an endogenous inhibitor of phosphodiesterase activity. Arterioscler Thromb. Vasc. Biol. 30, 1998-2004. doi:10.1161/ ATVBAHA.110.209783

Cortese-Krott, M. M., Kuhnle, G. G. C., Dyson, A., Fernandez, B. O., Grman, M., Dumond, J. F., et al. (2015). Key bioactive reaction products of the $\mathrm{NO} / \mathrm{H} 2 \mathrm{~S}$ interaction are S/N-hybrid species, polysulfides, and nitroxyl. Proc. Natl. Acad. Sci. USA 112, E4651-E4660. doi:10.1073/pnas. 1509277112

De Koning, M. S. L. Y., Assa, S., Maagdenberg, C. G., Van Veldhuisen, D. J., Pasch, A., Van Goor, H., et al. (2020). Safety and tolerability of sodium thiosulfate in patients with an acute coronary syndrome undergoing coronary angiography: a 
dose-escalation safety pilot study (SAFE-ACS). J. Interv. Cardiol. 2020, 1. doi:10.1155/2020/6014915

Drazner, M. H. (2011). The progression of hypertensive heart disease. Circulation 123, 327-334. doi:10.1161/CIRCULATIONAHA.108.845792

Erkens, R., Suvorava, T., Sutton, T. R., Fernandez, B. O., Mikus-Lelinska, M., Barbarino, F., et al. (2018). Nrf2 deficiency unmasks the significance of nitric oxide synthase activity for cardioprotection. Oxidative Med. Cell Longevity 2018, 1. doi:10.1155/2018/8309698

Fu, M., Zhang, W., Wu, L., Yang, G., Li, H., and Wang, R. (2012). Hydrogen sulfide $(\mathrm{H} 2 \mathrm{~S})$ metabolism in mitochondria and its regulatory role in energy production. Proc. Natl. Acad. Sci. 109, 2943-2948. doi:10.1073/pnas. 1115634109

Grafe, M., Bossaller, C., Graf, K., Auch-Schwelk, W., Baumgarten, C. R., Hildebrandt, A., et al. (1993). Effect of angiotensin-converting-enzyme inhibition on bradykinin metabolism by vascular endothelial cells. Am. J. Physiol.-Heart Circ. Physiol. 264, H1493-H1497. doi:10.1152/ajpheart. 1993.264.5.H1493

Huang, J., Wang, D., Zheng, J., Huang, X., and Jin, H. (2012). Hydrogen sulfide attenuates cardiac hypertrophy and fibrosis induced by abdominal aortic coarctation in rats. Mol. Med. Rep. 5, 923-928. doi:10.3892/mmr.2012.748

Huang, P., Shen, Z., Liu, J., Huang, Y., Chen, S., Yu, W., et al. (2016). Hydrogen sulfide inhibits high-salt diet-induced renal oxidative stress and kidney injury in dahl rats. Oxid. Med. Cell Longev. 2016, 1. doi:10.1155/2016/2807490

Hunt, G. M., and Ryder, H. F. (2018). Metabolic acidosis after sodium thiosulfate infusion and the role of hydrogen sulfide. Clin. Case Rep. 6, 1595-1599. doi:10. $1002 / \mathrm{ccr} 3.1673$

Jiang, B., Tang, G., Cao, K., Wu, L., and Wang, R. (2010). Molecular mechanism for H2S-induced activation of KATPChannels. Antioxid. Redox Signaling 12, 1167-1178. doi:10.1089/ars.2009.2894

Jin, S., Teng, X., Xiao, L., Xue, H., Guo, Q., Duan, X., et al. (2017). Hydrogen sulfide ameliorated L-NAME-induced hypertensive heart disease by the Akt/eNOS/ NO pathway. Exp. Biol. Med. (Maywood) 242, 1831-1841. doi:10.1177/ 1535370217732325

Kang, S. C., Sohn, E. H., and Lee, S. R. (2020). Hydrogen sulfide as a potential alternative for the treatment of myocardial fibrosis. Oxid. Med. Cell Longev. 2020, 1. doi:10.1155/2020/4105382

Kimura, Y., Goto, Y. I., and Kimura, H. (2010). Hydrogen sulfide increases glutathione production and suppresses oxidative stress in mitochondria. Antioxid. Redox Signaling 12, 1-13. doi:10.1089/ars.2008.2282

Kjeldsen, S. E. (2018). Hypertension and cardiovascular risk: general aspects. Pharmacol. Res. 129, 95-99. doi:10.1016/j.phrs.2017.11.003

Kobayashi, N., Hara, K., Watanabe, S., Higashi, T., and Matsuoka, H. (2000). Effect of imidapril on myocardial remodeling in L-NAME-induced hypertensive rats is associated with gene expression of NOS and ACE mRNA. Am. J. Hypertens. 13, 199-207. doi:10.1016/s0895-7061(99)00117-x

Koeners, M. P., Braam, B., and Joles, J. A. (2011). Perinatal inhibition of NFkappaB has long-term antihypertensive effects in spontaneously hypertensive rats. J. Hypertens. 29, 1160-1166. doi:10.1097/HJH.0b013e3283468344

Koitabashi, N., and Kass, D. A. (2011). Reverse remodeling in heart failuremechanisms and therapeutic opportunities. Nat. Rev. Cardiol. 9, 147-157. doi:10.1038/nrcardio.2011.172

Laggner, H., Hermann, M., Esterbauer, H., Muellner, M. K., Exner, M., Gmeiner, B. M., et al. (2007). The novel gaseous vasorelaxant hydrogen sulfide inhibits angiotensin-converting enzyme activity of endothelial cells. J. Hypertens. 25, 2100-2104. doi:10.1097/HJH.0b013e32829b8fd0

Li, N., Wang, M.-J., Jin, S., Bai, Y.-D., Hou, C.-L., Ma, F.-F., et al. (2016). The H2S donor NaHS changes the expression pattern of H2S-producing enzymes after myocardial infarction. Oxid. Med. Cell Longev. 2016, 1. doi:10.1155/2016/ 6492469

Liu, Y. H., Lu, M., Hu, L. F., Wong, P. T. H., Webb, G. D., and Bian, J. S. (2012). Hydrogen sulfide in the mammalian cardiovascular system. Antioxid. Redox Signaling 17, 141-185. doi:10.1089/ars.2011.4005

Lu, M., Liu, Y. H., Goh, H. S., Wang, J. J. X., Yong, Q.-C., Wang, R., et al. (2010). Hydrogen sulfide inhibits plasma renin activity. J. Am Soc. Nephrol. 21, 993-1002. doi:10.1681/ASN.2009090949

Meng, G., Ma, Y., Xie, L., Ferro, A., and Ji, Y. (2015). Emerging role of hydrogen sulfide in hypertension and related cardiovascular diseases. Br. J. Pharmacol. 172, 5501-5511. doi:10.1111/bph.12900
Mustafa, A. K., Sikka, G., Gazi, S. K., Steppan, J., Jung, S. M., Bhunia, A. K., et al. (2011). Hydrogen sulfide as endothelium-derived hyperpolarizing factor sulfhydrates potassium channels. Circ. Res. 109, 1259-1268. doi:10.1161/ CIRCRESAHA.111.240242

Nabeebaccus, A. A., and Shah, A. M. (2018). Hydrogen sulfide therapy promotes beneficial systemic effects in experimental heart failure. JACC: Basic Transl. Sci. 3, 810-812. doi:10.1016/j.jacbts.2018.11.009

Nguyen, I. T. N., Klooster, A., Minnion, M., Feelisch, M., Verhaar, M. C., Van Goor, H., et al. (2020). Sodium thiosulfate improves renal function and oxygenation in L-NNA-induced hypertension in rats. Kidney Int. 98, 366-377. doi:10.1016/j.kint.2020.02.020

Olson, K. R. (2011). The therapeutic potential of hydrogen sulfide: separating hype from hope. Am. J. Physiol.-Regul., Integr. Comp. Physiol. 301, R297-R312. doi:10.1152/ajpregu.00045.2011

Pan, L. L., Qin, M., Liu, X. H., and Zhu, Y. Z. (2017). The role of hydrogen sulfide on cardiovascular homeostasis: an overview with update on immunomodulation. Front. Pharmacol. 8, 686. doi:10.3389/fphar.2017.00686

Pan, L. L., Wang, X. L., Wang, X. L., and Zhu, Y. Z. (2014). Sodium hydrosulfide prevents myocardial dysfunction through modulation of extracellular matrix accumulation and vascular density. Int. J. Mol. Sci. 15, 23212-23226. doi:10. 3390/ijms151223212

Papazova, D. A., Krebber, M. M., Oosterhuis, N. R., Gremmels, H., Van Zuilen, A. D., Joles, J. A., et al. (2018). Dissecting recipient from donor contribution in experimental kidney transplantation: focus on endothelial proliferation and inflammation. Dis. Model. Mech. 11, dmm035030. doi:10.1242/dmm.035030

Papazova, D. A., Van Koppen, A., Koeners, M. P., Bleys, R. L., Verhaar, M. C., and Joles, J. A. (2014). Maintenance of hypertensive hemodynamics does not depend on ROS in established experimental chronic kidney disease. PLoS One 9, e88596. doi:10.1371/journal.pone.0088596

Perridon, B. W., Leuvenink, H. G. D., Hillebrands, J.-L., Van Goor, H., and Bos, E. M. (2016). The role of hydrogen sulfide in aging and age-related pathologies. Aging 8, 2264-2289. doi:10.18632/aging.101026

Powell, C. R., Dillon, K. M., and Matson, J. B. (2018). A review of hydrogen sulfide (H2S) donors: chemistry and potential therapeutic applications. Biochem. Pharmacol. 149, 110-123. doi:10.1016/j.bcp.2017.11.014

Racasan, S., Braam, B., Van Der Giezen, D. M., Goldschmeding, R., Boer, P., Koomans, H. A., et al. (2004). Perinatall 1 -arginine and antioxidant supplements reduce adult blood pressure in spontaneously hypertensive rats. Hypertension 44, 83-88. doi:10.1161/01.HYP.0000133251.40322.20

Rein, J. L., Miyata, K. N., Dadzie, K. A., Gruber, S. J., Sulica, R., and Winchester, J. F. (2014). Successfully treated calcific uremic arteriolopathy: two cases of a high anion gap metabolic acidosis with intravenous sodium thiosulfate. Case Rep. Nephrol. 2014, 1. doi:10.1155/2014/765134

Sanada, S., Kitakaze, M., Node, K., Takashima, S., Ogai, A., Asanuma, H., et al. (2001). Differential subcellular actions of ACE inhibitors and at 1 receptor antagonists on cardiac remodeling induced by chronic inhibition of NO synthesis in rats. Hypertension 38, 404-411. doi:10.1161/01.hyp.38.3.404

Schulz, E., Gori, T., and Münzel, T. (2011). Oxidative stress and endothelial dysfunction in hypertension. Hypertens. Res. 34, 665-673. doi:10.1038/hr. 2011.39

Sen, U., Vacek, T. P., Hughes, W. M., Kumar, M., Moshal, K. S., Tyagi, N., et al. (2008). Cardioprotective role of sodium thiosulfate on chronic heart failure by modulating endogenous H2S generation. Pharmacology 82, 201-213. doi:10. $1159 / 000156486$

Shen, Y., Shen, Z., Luo, S., Guo, W., and Zhu, Y. Z. (2015). The cardioprotective effects of hydrogen sulfide in heart diseases: from molecular mechanisms to therapeutic potential. Oxidative Med. Cell Longevity 2015, 1. doi:10.1155/2015/ 925167

Singh, R. P., Derendorf, H., and Ross, E. A. (2011). Simulation-based sodium thiosulfate dosing strategies for the treatment of calciphylaxis. Clin. J. Am. Soc. Nephrol. 6, 1155-1159. doi:10.2215/CJN.09671010

Snijder, P. M., Frenay, A. R. S., Koning, A. M., Bachtler, M., Pasch, A., Kwakernaak, A. J., et al. (2014). Sodium thiosulfate attenuates angiotensin II-induced hypertension, proteinuria and renal damagel1These authors contributed equally to this manuscript. Nitric Oxide 42, 87-98. doi:10.1016/j.niox.2014. 10.002

Snijder, P. M., Frenay, A. R., De Boer, R. A., Pasch, A., Hillebrands, J. L., Leuvenink, H. G. D., et al. (2015). Exogenous administration of thiosulfate, a donor of 
hydrogen sulfide, attenuates angiotensin II-induced hypertensive heart disease in rats. Br. J. Pharmacol. 172, 1494-1504. doi:10.1111/bph.12825

Sowers, K. M., and Hayden, M. R. (2010). Calcific uremic arteriolopathy: pathophysiology, reactive oxygen species and therapeutic approaches. Oxidative Med. Cell Longevity 3, 109-121. doi:10.4161/oxim.3.2.11354

Tamura, T., Said, S., Harris, J., Lu, W., and Gerdes, A. M. (2000). Reverse remodeling of cardiac myocyte hypertrophy in hypertension and failure by targeting of the renin-angiotensin system. Circulation 102, 253-259. doi:10. 1161/01.cir.102.2.253

Verhagen, A. M. G., Braam, B., Boer, P., Gröne, H.-J., Koomans, H. A., and Joles, J. A. (1999). Losartan-sensitive renal damage caused by chronic NOS inhibition does not involve increased renal angiotensin II concentrations. Kidney Int. 56, 222-231. doi:10.1046/j.1523-1755.1999.00542.x

Verhagen, A. M. G., Hohbach, J., Joles, J. A., Braam, B., Boer, P., Koomans, H. A., et al. (2000). Unchanged cardiac angiotensin II levels accompany losartansensitive cardiac injury due to nitric oxide synthase inhibition. Eur. J. Pharmacol. 400, 239-247. doi:10.1016/s0014-2999(00)00384-8

Wang, R. (2010). Hydrogen sulfide: the third gasotransmitter in biology and medicine. Antioxid. Redox Signaling 12, 1061-1064. doi:10.1089/ars.2009.2938

Wang, X., Wang, Q., Guo, W., and Zhu, Y. Z. (2011). Hydrogen sulfide attenuates cardiac dysfunction in a rat model of heart failure: a mechanism through cardiac mitochondrial protection. Biosci. Rep. 31, 87-98. doi:10.1042/ BSR20100003

Whiteman, M., and Moore, P. K. (2009). Hydrogen sulfide and the vasculature: a novel vasculoprotective entity and regulator of nitric oxide bioavailability? J. Cell Mol. Med. 13, 488-507. doi:10.1111/j.1582-4934.2009.00645.x
Xiao, L., Dong, J.-H., Jin, S., Xue, H.-M., Guo, Q., Teng, X., et al. (2016). Hydrogen sulfide improves endothelial dysfunction via downregulating BMP4/COX-2 pathway in rats with hypertension. Oxid. Med. Cell Longev. 2016, 1. doi:10. $1155 / 2016 / 8128957$

Yan, H., Du, J., and Tang, C. (2004). The possible role of hydrogen sulfide on the pathogenesis of spontaneous hypertension in rats. Biochem. Biophysical Res. Commun. 313, 22-27. doi:10.1016/j.bbrc.2003.11.081

Zhao, W., Zhang, J., Lu, Y., and Wang, R. (2001). The vasorelaxant effect of H2S as a novel endogenous gaseous KATP channel opener. EMBO J. 20, 6008-6016. doi:10.1093/emboj/20.21.6008

Zhong, G., Chen, F., Cheng, Y., Tang, C., and Du, J. (2003). The role of hydrogen sulfide generation in the pathogenesis of hypertension in rats induced by inhibition of nitric oxide synthase. J. Hypertens. 21, 1879-1885. doi:10.1097/ 00004872-200310000-00015

Conflict of Interest: The authors declare that the research was conducted in the absence of any commercial or financial relationships that could be construed as a potential conflict of interest.

Copyright $\odot 2021$ Nguyen, Wiggenhauser, Bulthuis, Hillebrands, Feelisch, Verhaar, van Goor and Joles. This is an open-access article distributed under the terms of the Creative Commons Attribution License (CC BY). The use, distribution or reproduction in other forums is permitted, provided the original author(s) and the copyright owner(s) are credited and that the original publication in this journal is cited, in accordance with accepted academic practice. No use, distribution or reproduction is permitted which does not comply with these terms. 